Cahiers de philosophie de l'université de

Levinas : au-delà du visible

\title{
En chemin vers le sens du langage
}

\section{Francis Guibal}

\section{(2) OpenEdition}

Journals

Édition électronique

URL : https://journals.openedition.org/cpuc/837

DOI : 10.4000/cpuc.837

ISSN : 2677-6529

Éditeur

Presses universitaires de Caen

\section{Édition imprimée}

Date de publication : 31 juillet 2012

Pagination : 161-178

ISBN : 978-2-84133-410-0

ISSN : 1282-6545

\section{Référence électronique}

Francis Guibal, «En chemin vers le sens du langage », Cahiers de philosophie de l'université de Caen [En ligne], 49 | 2012, mis en ligne le 07 juin 2018, consulté le 01 février 2023. URL : http:// journals.openedition.org/cpuc/837 ; DOI : https://doi.org/10.4000/cpuc.837

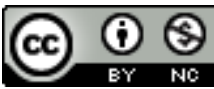

Creative Commons - Attribution - Pas d'Utilisation Commerciale 4.0 International - CC BY-NC 4.0 https://creativecommons.org/licenses/by-nc/4.0/ 


\section{En chemin vers le sens du langage}

Parler n'est-ce pas originellement écouter?

Levinas $^{1}$

\section{$\mathrm{L}$} ES PREMIERS TEXTES PUBLIÉS par Emmanuel Levinas portent à l'évidence la marque d'un «monde cassé», sous le signe de la pesanteur anonyme d'un «il y a » ne pouvant susciter qu'un besoin «d'évasion» apparemment voué à l'échec. À cette opacité répond très tôt, cependant, la gravité d'un êtrerivé-au-judaïsme où s'atteste l'irruption d'un hors-monde faisant éclater les limites de l'immanence païenne. Ce qui conduit, philosophiquement, à se demander si l'intentionnalité qui ordonne la conscience à l'accueil du dehors relève bien d'une simple donation de sens à la stricte mesure d'un sujet qui «répond toujours de lui-même et à lui-même $»^{2}$. L'expérience de la captivité approfondira ces interrogations en les prolongeant notamment du côté de la sexualité ou de l'esthétique ${ }^{3}$. Mais ce sont évidemment les textes des années 1947-1948 qui témoignent d'une problématique originale qui commence à se mettre en place.

La nouveauté, dans les essais de cette époque, se dit notamment à travers l'instance de ce sujet «substantiel» qu'est l'hypostase: position ou tenue

1. Notes philosophiques diverses, liasse B, in Carnets de captivité, suivi de Écrits sur la captivité et Notes philosophiques diverses (= Euvres 1), R. Calin et C. Chalier (éd.), Paris, Grasset IMEC, 2009, p. 376.

2. «L'œuvre de Edmund Husserl», article de 1940, repris dans En découvrant l'existence avec Husserl et Heidegger, $2^{\mathrm{e}}$ éd., Paris, Vrin, 1967, p. 7-52; citation p. 47.

3. Les Carnets de captivité pensent la sexualité « comme origine du social» (Carnets de captivité, in CEuvres 1, carnet 2, p. 66), "constitutive de l'égoïté» (carnet 4, p. 114). Le rapport des deux se noue dans une « dialectique sexuelle» (carnet 5, p. 142). Et le même carnet 5 semble s'orienter vers une conception artistique de la métaphysique et de l'existence: «La dernière conséquence de ma conception esthétique - la métaphysique est en fin de compte un art, le sens de l'existence est un art? - l'existence un art?» (p. 132). 
d'un soi séparé et solitaire, mais dans une «instantanéité» qui s'essouffle inévitablement à s'arracher à l'engluement naturel. Pour échapper, dès lors, au malheur de cette «fatalité dans laquelle se fige la liberté» ${ }^{4}$, c'est à la merveille mystérieuse de la rencontre sexuelle et au temps discontinu de la fécondité érotique que Levinas en appelle: le «salut» ne devrait-il pas être cherché dans la pluralité sans fin recommencée des générations? Sans que soit encore vraiment prise en compte la dimension proprement langagière des rapports interhumains.

Celle-ci commence pourtant à apparaître, négativement, à travers la critique sévère du "commerce avec l'obscur ${ }^{5}$ qui se joue dans l'imaginaire artistique. Faute de langage critique susceptible de "mettre en mouvement et de faire parler» ses statues immobiles, la figuration plastique "lâche la proie pour l'ombre» et se voue à la mystification caricaturale d'une stupidité idolâtrique; aussi Levinas dénonce-t-il avec vigueur l'hypertrophie moderne de l'art et son identification abusive à «la vie spirituelle», cependant qu'il lui oppose une "proscription des images» qui «est véritablement le suprême commandement du monothéisme, d'une doctrine qui surmonte le destin " grâce au recours à la transcendance de la parole ${ }^{6}$. Mais cela ne suffit pas pour donner forme de cohérence raisonnablement argumentée à ce qui relève d'abord d'une sorte d'intuition religieuse; un long chemin de pensée sépare encore Levinas des conclusions magistralement élaborées et exposées dans Totalité et Infini.

\section{La gloire ambiguë de la créature}

Alors même qu'elle médite sur la grandeur du langage et la dignité de l'être parlant, la conférence de 1948 sur «Parole et Silence» accorde, de manière significative, une place importante à ce que présuppose cette émergence originale ${ }^{7}$. Pour situer et évaluer philosophiquement l'ouverture qui se fait jour à travers cette venue à soi de l'animal qui a le logos en partage, il faut bien partir du sol d'expérience qui la porte et la rend possible. La position dans le monde de la vie de corps animés, dotés d'intériorité psychique, dans lesquels participation et séparation s'entrelacent de manière paradoxale,

4. De l'existence à l'existant [1947], ${ }^{\mathrm{e}}$ éd., Paris, Vrin, 1978, p. 133.

5. "La réalité et son ombre», texte de 1948 repris dans Les imprévus de l'histoire, Montpellier, Fata Morgana, 1994, p. 123-148; citation p. 126.

6. Voir «La réalité et son ombre», p. 143-148.

7. «Parole et Silence», in E. Levinas, Parole et Silence et autres conférences inédites au Collège philosophique (= Euvres 2), R. Calin et C. Chalier (éd.), Paris, Grasset - IMEC, 2011, p. 65-104. 
voilà sans doute ce que doit d'abord s'attacher à décrire une phénoménologie soucieuse de ne pas séparer le sens de son ancrage sensible ${ }^{8}$.

On ne s'étonnera donc pas que Levinas reparte de ses descriptions antérieures de l'hypostase et de son «in-sistance», mais en attribuant désormais une qualification et comme une coloration «glorieuses» à cette "position» qui caractérise «l'être chez soi »9. Si la gloire peut être définie comme un "poids d'être par lequel l'être s'affirme $»^{10}$, elle se donne à éprouver par la subjectivité finie comme une «assise dans l'être» qui «s'oppose à la honte d'être ${ }^{11}$ en ce qu'elle atteste «le caractère créé du sujet ${ }^{12}$ et la "sécurité d'un monde créé ${ }^{13}$. Tout se passe comme si la merveille «miraculeuse ${ }^{14}$ de l'existence libérait l'existant fini de sa crispation obsessionnelle sur soi et ses "pouvoirs» en lui faisant découvrir «l'origine comme sécurité » imprenable s'étendant à tout le monde de la vie et de sa fécondité.

Même s'il se donne dans la lumière d'une immanence qui est aussi «sa mise à ma disposition [...] dans sa présentation même ${ }^{16}$, ce monde n’a rien ni d'un spectacle théorique ni d'une instrumentalité pratique. Son immédiateté généreuse est celle d'une profusion sensible radicalement distincte, notamment, de "l'ensemble des ustensiles aboutissant au souci d'une existence angoissée de sa mort ${ }^{17}$. Et l'intentionnalité du corps de besoins qui se porte vers cette réalité donnée, à laquelle il appartient et dont il vit, ne relève d'aucune maîtrise, constituante ou existentiale, mais d'un simple «mouvement naïf vers les choses qui nourrissent la vie, vers la jouissance des nourritures ${ }^{18}$. $\mathrm{Si}$ « sagesse $»$ il y a ici, c'est celle d'un animal

8. Levinas fera toujours droit à cet enracinement de la pensée du sens dans la concrétude du sensible; dans Totalité et Infini (La Haye, Martinus Nijhoff, 1961), cela s'indique notamment à travers le rapport des deux sections centrales, «Le visage et l'extériorité» ne survenant que sur fond d'«intériorité et économie».

9. "Parole et Silence», p. 88.

10. Ibid., p. 89 .

11. Notes philosophiques diverses, liasse C, p. 448.

12. "Parole et Silence», p. 95.

13. Ibid., p. 102. La conférence de 1949 sur « Pouvoirs et Origine» (Euvres 2, p. 105-150) évoque «la sécurité même d'un être dont l'exister se réfère à l'événement de la fécondité: la sécurité de la créature» (p. 140) moins «jetée» dans l'existence que suscitée et posée en elle par une origine s'y donnant à éprouver comme parole créatrice.

14. Voir Carnets de captivité, carnet 7, p. 178.

15. "Pouvoirs et Origine», p. 137.

16. "Les Nourritures» [1950], in Euvres 2, p. 151-172; citation p. 159.

17. Ibid., p. 161. Dès 1948, Le Temps et l'Autre (Paris, PUF, 1983) notait, en opposition à Heidegger, «qu'avant d'être un système d'outils, le monde est un ensemble de nourritures» (p. 45). Totalité et Infini reprendra et développera (p. 100-108) ce sens original de la nourriture qui «ne peut s'interpréter comme ustensile que dans un monde d'exploitation» (p. 108).

18. «Les Nourritures», p. 162. 
humain sachant "goûter à la vie ${ }^{19}$ dans toute l'innocence pré-réflexive d'un accord savouré et savoureux.

Cet accord, cependant, n'est pas identité. Le corps qui vit du monde et en jouit est aussi celui qui se pose et se tient en lui et devant lui, qui est capable d'y établir sa demeure. Cette dualité de l'appartenance et de l'émergence indique donc bien déjà certaine "transcendance dans l'immanence ${ }^{20}$ : «Pouvoir accueillir le monde tout en faisant partie du monde, c'est être chez soi ${ }^{21}$. Mais la transcendance de ce "chez soi» n'est jamais que relative, elle ne cesse de prendre appui sur ce dont elle provient: «Le monde que je constitue est un monde dont je vis et où je vis ${ }^{22}$. Si «souveraineté» il $\mathrm{y}$ a, lisible dans la manière dont le corps se tient chez soi dans le monde qu'il habite, elle s'appuie toujours sur une «dépendance» qui donne à son «bonheur essentiellement égoïste ${ }^{23}$ la fragilité ou l'instabilité d'une insouciance inévitablement guettée par l'inquiétude du lendemain. La "position» de l'hypostase ne va jamais sans un "poids qui complique son voyage d'existence», grevant de fatigue et d'essoufflement «la résurrection de l'irremplaçable instant ${ }^{24}$.

Quoi qu'il en soit de cette dualité inhérente à la finitude de l'existant, elle ne doit pas masquer qu'apparaît ainsi une manière d'intériorité ou de psychisme caractérisée par une «séparation » qui «rompt la totalité» ${ }^{25}$. Un espace d'intimité secrète se creuse au cœur du monde de la vie: position à la fois singulière et plurielle de vivants distincts ou séparés, qui résistent à toute intégration dans un système de totalité homogène. Ce qui peut s'exprimer de manière paradoxale en parlant d'une "totalité d'êtres extérieurs à la totalité ${ }^{26}$. Chacun - chaque un - s'éprouvant ainsi livré, remis

19. Notes philosophiques diverses, liasse A, p. 245.

20. À propos de Husserl, dans «Réflexions sur la "technique" phénoménologique» [1959], article repris dans En découvrant l'existence avec Husserl et Heidegger, p. 111-123; citation p. 120 .

21. "Les Nourritures», p. 166.

22. «Le Vouloir» [1955], in Euvres 2, p. 231-257; citation p. 240. L'article de 1959 sur «La ruine de la représentation» reprendra cet entrelacs de la constitution et de l'appartenance ou de la liberté et de la condition: «l'homme constitue le monde auquel cependant déjà il appartient» (En découvrant l'existence avec Husserl et Heidegger, p. 125-135; citation p. 133). Et Totalité et Infini le redira: «Le monde que je constitue me nourrit et me baigne» (p. 102).

23. "dépendance qui vire en souveraineté, en bonheur essentiellement égoïste», cette belle formule de l'article de 1957 sur «La Séparation» (in Euvres 2, p. 259-289; citation p. 276) est reprise littéralement par Totalité et Infini (p. 87).

24. De l'existence à l'existant, p. 38 et 158. Totalité et Infini, là encore, n'éludera pas le paradoxe: «la possession de soi devient encombrement de soi» (p. 248).

25. "La Séparation", p. 265.

26. «Le Moi et la Totalité», texte de 1954, repris dans Entre nous, essais sur le penser-à-l'autre, Paris, Grasset, 1991, p. 25-52; citation p. 29. Cela invite à se demander s'il ne faut pas distinguer entre diverses formes de «totalité»... 
ou abandonné à "lui-même» (ipséité), dans une «séparation si complète que l'être séparé se maintient tout seul dans l'existence » ${ }^{27}$ et qu'il peut être dit radicalement athée ${ }^{28}$. Levinas ne cessera guère de méditer sur cette merveille énigmatique d'une création comme éclatée en existants pluriels rompant toute participation onto-théo-logique par l'absoluité de leur indépendance: «On peut appeler créature un être qui par la volonté affirme l'indépendance de l'existence dont il n'est pas l'origine. [...] La créature ne consiste pas à avoir une cause extérieure à soi, mais à être indépendant dans cette dépendance ${ }^{29}$.

Que peuvent signifier ces préalables pour une approche phénoménologique du langage? La reconnaissance, tout d'abord, que ce dernier n'est pas une entité absolue, qu'il ne peut naître que sur le sol d'une vie originale, inséparablement corporelle et psychique, qui s'éprouve déjà rebelle aux intégrations totalisantes. Mais la mise en évidence, également, de l'ambiguité constitutive de cette innocence première, susceptible aussi bien de s'ériger en clôture narcissique que de s'ouvrir à l'interpellation du dehors. La "gloire» de la créature indépendante, en effet, tend inévitablement à virer en «fatalité ${ }^{30}$ païenne ou idolâtre lorsqu'elle se fixe dans la solitude égoïste d'une ipséité non concernée par autrui ${ }^{31}$. Et le recours à la fécondité de l'amour pourrait bien être une voie trop courte pour sortir de l'égologie s'il est vrai que la sphère de l'intimité est « réfractaire à l'universalité» et "rend aveugle le respect» ${ }^{32}$. Il n'en demeure pas moins que cette gloire de l'ipséité séparée est la "condition de la parole» ${ }^{33}$, qu'elle seule rend la créature capable d' «entendre ${ }^{34}$, «capable de questions et de réponses,

27. «La Séparation", p. 272. Totalité et Infini retiendra la formule, que ce soit pour la présence à soi du cogito (p. 25) ou pour l'athéisme entendu comme «séparation si complète que l'être séparé se maintient tout seul dans l'existence sans participer à l'Être dont il est séparé» (p. 29).

28. Dans ses Notes philosophiques diverses, Levinas évoque le «séparé-athée» (liasse A, p. 256). Totalité et Infini précisera : «L'âme - la dimension du psychisme - accomplissement de la séparation, est naturellement athée» (p. 29).

29. Notes philosophiques diverses, liasse A, p. 257. Totalité et Infini dira que "c'est certainement une grande gloire pour le créateur d'avoir mis sur pieds un être capable d'athéisme [...], qui a le regard et la parole indépendants et qui est chez soi» (p. 30); en précisant d'ailleurs que cet athéisme qualifie une "position antérieure à la négation comme à l'affirmation du divin» (ibid.).

30. "Parole et Silence», p. 96.

31. Cf. la p. 179 de l'essai sur «Les Enseignements» [1950], in Euvres 2, p. 173-198.

32. «Le Moi et la Totalité», p. 33, qui n’hésite pas à affirmer, en ce sens, que «la loi prime la charité» (p. 35) pour l'animal politique qu'est l'homme.

33. Notes philosophiques diverses, liasse A, p. 257.

34. «Parole et Silence», p. 89. 
responsable ${ }^{35}$, apte donc à jouer « un rôle d'interlocuteur ${ }^{36}$. Il faut des corps qui se tiennent déjà "dressé(s) de bas vers le haut» ${ }^{37}$ pour se laisser ouvrir à la grâce de la bénédiction.

\section{Le langage économique des significations}

Cette insistance première sur l'originalité du psychisme et de son intériorité n'est donc pas dupe de l'abstraction qu'elle opère. Le monde de la vie, en effet, est déjà "plein de significations ${ }^{38}$ immanentes et, à vrai dire, l'homme $\mathrm{y}$ a depuis toujours "reçu des enseignements» ${ }^{39}$. Le donné sensible peut bien s'offrir comme nourriture à la jouissance, il n'échappe pas à une mise en perspective, voire à une mise en ordre, qui le situe dans le monde et qui est le fait du langage et de la culture. Comment s'effectue cette assomption et rend-elle vraiment justice à ce qui se propose ainsi à elle? La question invite à prendre la mesure des traditions qui nous portent et des orientations philosophiques qui en dégagent la cohérence.

De manière assez classique, Levinas définit d'abord le langage comme un « système de signes » qui « actualise la raison en chacun » ${ }^{\circ}$ des interlocuteurs. Cette actualisation raisonnable s'exerce notamment face à la violence du chaos et à l'arbitraire des individus: le logos impose à tout et à tous la loi de son ordre. Les significations qu'il déploie opposent «au monde nocturne de l'il y a [...] le monde de la lumière ${ }^{41}$. Il ne s'agit pas tant de contempler la cohérence d'un monde donné que de forger un réseau de relations permettant de saisir "ceci en tant que cela» ${ }^{42}$ à l'intérieur d'un ensemble contextuel où "se ramasse pour s'éclairer une totalité d'expérience » ${ }^{43}$

35. Notes philosophiques diverses, liasse A, p. 257.

36. "La Séparation ", p. 273. Les Notes philosophiques diverses soulignent le lien entre sortie du système et accès à la parole (liasse A, p. 257). Les articles de 1959 sur «La technique phénoménologique» et «La ruine de la représentation» vont dans le même sens: seul un sujet «arraché à totalité» est capable de "parler», ouvert donc à la possibilité d'une «Sinngebung éthique, c'est-dire essentiellement respectueuse de l'Autre» (En découvrant l'existence avec Husserl et Heidegger, p. 121 et 135). Et Totalité et Infini le redira: «La transcendance est transcendance d'un moi. Seul un moi peut répondre à l'injonction d'un visage» (p. 282).

37. Totalité et Infini, p. 89.

38. «Les Nourritures», p. 168.

39. Ibid., p. 164.

40. "Parole et Silence», p. 75.

41. Ibid., p. 79.

42. "La Métaphore» [1962], in Euvres 2, p. 319-347; citation p. 334.

43. "La Signification et le Sens", article de 1964 repris dans Humanisme de l'autre homme, Montpellier, Fata Morgana, 1972, p. 17-63; citation p. 23, renvoyant à Heidegger. Ce grand article reprend nombre d'éléments élaborés dans les inédits, notamment «La Métaphore» et «La Signification» (Euvres 2, p. 349-382); je me permets de renvoyer au commentaire que j'en ai proposé dans le chapitre «Hauteur: cultures et transcendance» de mon ouvrage Emmanuel Levinas ou les intrigues du sens, Paris, PUF, 2005, p. 79-115. 
signifiante. Dans et par ce milieu d'intelligibilité qu'ils mettent en place, les sujets parlants se rendent maîtres de l'être " qui se révèle à la raison " ${ }^{44}$. N'est-ce pas l'éminente dignité de l'animal humain qui se trouve attestée par cette manière de porter la réalité à la lumière du sens 45 ?

De fait, c'est bien grâce à la cohérence du langage que les hommes donnent au monde forme d'homogénéité rationnelle, cependant qu'euxmêmes se laissent éduquer par les échanges universalisants du dialogue raisonnable. Mais il faut mesurer le prix d'une telle mise en ordre systématique. Il semble bien, en effet, que l'extériorité n'accède ainsi à la pensée qu'en se laissant réduire à la condition abstraite d'une objectivité vidée de toute richesse sensible. Quant aux sujets de cette réduction intelligible, ils paraissent condamnés à renoncer à leur originalité singulière et à s'effacer "dans l'impersonnalité de la raison» ${ }^{46}$ qui soumet tout à la "tyrannie de l'universalité» ${ }^{47}$. Que la souveraineté qui s'impose de ce fait soit celle de l'Esprit dans son histoire ou de l'Être dans ses "époques", il n'importe d'une certaine manière. Le langage qui établit ce règne est "discours sans interlocuteurs, car les interlocuteurs eux-mêmes y figurent comme "moments"» 48 .

L'histoire, pourtant, tend à relativiser ces prétentions spéculatives et inviter à prendre une conscience plus modeste et plus critique des explorations signifiantes du langage. Au lieu d'être trop superbement référé aux avancées d'un absolu ontologique ou spirituel, ne doit-il pas plutôt être reconnu comme l'expression esthétique et poétique de corps vivants qui «célèbrent» la beauté mystérieuse du monde par leurs gestes et leurs danses, leurs chants et leurs échanges? Et ce langage, d'ailleurs, dans la mesure même où il est parlé par des "esprits incarnés", ne s'éprouve-t-il pas immédiatement fragmenté en langues culturelles irrémédiablement diverses? «La signification culturelle» alors peut bien être pensée comme «l'ultime sens de la signification» ${ }^{49}$, elle n'a d'effectivité que disséminée en éclats de sens où s'atteste en scintillements multiples l’inépuisable richesse du monde sensible.

\footnotetext{
44. «Parole et Silence», p. 76.

45. «La Signification et le Sens » évoquera cette structure signifiante du monde ordonné par le langage en parlant de la «totalité de l'être où l'être resplendit comme signification» (p. 30).

46. "Parole et Silence», p. 81.

47. "Le Vouloir», p. 235.

48. «Le Moi et la Totalité», p. 37. Le terme de «moments» semble bien faire référence au discours hégélien qui absorbe ou résorbe les sujets parlants (voir Totalité et Infini, p. 192) dans un système qui serait liberté et raison ne laissant rien hors de soi et de son universalité concrète.
}

49. «La Signification», p. 365 . 
Voilà qui permet de reconnaître la signification positive, mais relative, de l'action qui peut être dite de "civilisation" et du "monde des œuvres " ${ }^{\circ}$ qu'elle institue. Tout se passe comme si la vie fragile de la créature ne cessait d'ajourner la menace de l'insécurité, du vieillissement et finalement de la mort «en se donnant toujours une distance à son égard ${ }^{51}$. Il y faut sans doute le travail tâtonnant d'une main qui « $v a$ à l'aventure " ${ }^{52}$, mais la métaphoricité constitutive du langage est aussi toujours déjà là pour articuler, guider et ordonner le sens de ce processus économique qui s'étend à la politique et à l'histoire tout en demeurant «à la mesure de la pensée " ${ }^{53}$. S'il paraît impossible de se passer des avancées civilisatrices de cette objectivation, on ne saurait se leurrer quant aux limites à l'intérieur desquelles elles opèrent. C'est qu'il ne suffit pas de passer de la domination conceptuelle à l'évocation métaphorique pour sortir véritablement de l'immanence ontologique, soit d'une totalitéen-mouvement où ne résonne jamais que «l'appel du semblable par le semblable " ${ }^{4}$. Ce monde de la relativité plurielle est bien celui de différences, de renvois, voire de transports et de dépassements à l'in(dé)fini, mais toujours à l'intérieur d'échanges "économiques" dans et entre les cultures. Les sujets qui s'y engagent n'y adviennent qu'absentés d'euxmêmes, sous des masques voués aux malentendus et à la désorientation d'une pseudo-reconnaissance.

Faut-il choisir entre la totalité systématique du discours monologique de l'être et la "totalité ouverte " 55 de la signifiance (inter)culturelle et de ses jeux multiples? Levinas soupçonne, quant à lui, certaine complicité entre des figures opposées du "même»: que la liberté s'ordonne à la souveraineté universelle de la raison ou qu'elle s'investisse dans les significations relatives des cultures, elle tend à se méconnaître elle-même en se soustrayant "également » à l'irruption et à l'interpellation impérative de l'Autre. Est-il possible de laisser venir à la pensée ce langage premier d'une altérité transcendante?

50. «Le Vouloir», p. 249-250.

51. Ibid., p. 251.

52. «La Séparation», p. 281.

53. Notes philosophiques diverses, liasse A, p. 331. Totalité et Infini dira de tout ce processus qu'il est «économique», c'est-à-dire qu'il «vient de la maison et y retourne» (p. 151).

54. Notes philosophiques diverses, liasse A, p. 257. En ce sens, note Levinas, «toute métaphore reste dans l'immanence» (ibid., p. 330) de renvois relationnels homogènes.

55. "Totalité et totalisation», article de l'Encyclopaedia Universalis, repris dans Altérité et Transcendance, Montpellier, Fata Morgana, 1995, p. 57-68; la "notion de totalité ouverte» (p. 66) caractériserait une «totalité herméneutique» distincte de la totalité spéculative. 


\section{La percée de la parole enseignante}

Dans ce qui se donne pour l'ordre normal et commun des phénomènes, le langage s'est montré dans sa fonction de mise en ordre intelligible. Et il ne faudra sans doute jamais oublier ce tissu relationnel qui préside à la cohérence relative des rapports sociaux et culturels des humains. Mais n'y a-t-il pas une autre dimension, plus originaire quoique moins apparente, de l'interlocution et de son aventure? Une expérience qui arracherait au(x) contexte(s) des prétendues évidences partagées et permettrait aux sujets parlants de remonter jusqu'au "principe» qui les juge en les relançant tout autrement? Au lieu des discours en quête de savoir assuré, la surprise impérative de l'interpellation...

Au logos lumineux de la théorie vient s'adjoindre, en ce sens, la vibration plus dérangeante du son entendu comme "éclat » et "retentissement", résonance et répercussion. Ce qui s'annonce par là, c'est «la gloire d'un autre être ${ }^{56}$ qui frappe à la porte et dont la survenue, imprévisible et fulgurante, oblige à prendre en compte «le mystérieux de l'être en tant qu'autre " 57 , en tant qu'ouvert à l'éventualité d'un autrement inanticipable. «La sonorité $[. .$.$] décrit la structure d'un monde où l'autre peut$ apparaître ${ }^{58}$ et déjouer les attentes du «même».

Ainsi lié à la voix qui le porte, le langage fait exploser les limites de la conscience enfermée dans l'autonomie de son intériorité. Si son essence «est enseignement " 59 , ce n'est donc pas au sens de la fameuse «maïeutique» socratique. Ici, le «maître» et l'extériorité de sa parole vive résistent si bien à toute assimilation identifiante qu'ils obligent la pensée à se laisser porter hors d'elle-même par l'enseignement magistral qui lui est délivré. Il faut cette altérité du maître enseignant - «Son altérité est sa maîtrise» va jusqu'à dire Levinas ${ }^{60}$ - pour interpréter l'écriture des textes transmis en apprenant à écouter en eux les voix qui les suscitent et les traversent: «Enseigner, c'est remonter de la lettre à la parole, c'est apprendre à lire les écrits ${ }^{61}$ en leur puissance d'inspiration toujours à raviver.

56. «Parole et Silence», p. 90.

57. Ibid., p. 92.

58. Ibid., p. 93.

59. Ibid., p. 85 .

60. "Pouvoirs et Origine», p. 148. Totalité et Infini précisera que cette altérité magistrale est «le premier enseignement de la raison» et «la condition de tout enseignement» (p. 178) effectif.

61. «Pouvoirs et Origine», p. 147. La conférence de 1950 sur «Les Enseignements» (p. 173-198) précise ce sens de l'enseignement qui sera encore au cœur de Totalité et Infini: «L'enseignement signifie tout l'infini de l'extériorité» (p. 146). Je me permets de renvoyer au chapitre 1, «Autorité et éveil», de mon ouvrage Approches d'Emmanuel Levinas - L'inspiration d'une écriture, Paris, PUF, 2005, p. 17-34. 
Ce maître, cependant, n'énonce aucun contenu objectif et n'est pas concrètement identifiable. Ce qui parle en et par lui, étrangement, c'est l'altérité pure d'une intimation imprenable dont la résonance silencieuse passe sans se fixer à travers "l'indissimulable langage des yeux ${ }^{62}$. En cela même qu'elle "défait à tout instant la forme qu'elle offre " ${ }^{63}$, l'originalité singulièrement signifiante de cette auto-expression énigmatique résiste à toutes les appropriations intériorisantes et excède tous les contextes particuliers ${ }^{64}$. Levinas propose d'y reconnaître moins une expérience que l'expérience par excellence, «l'expérience au seul sens radical de ce terme: une relation avec l'extérieur, avec l'Autre, sans que cette extériorité puisse s'intégrer au Même» ${ }^{65}$. Ce qui « ouvre le discours » ${ }^{66}$ ici est moins la décision de la liberté que la provocation qui la questionne ${ }^{67}$.

Ce que Levinas nomme ainsi «langage originel ${ }^{68}{ }^{\prime}$ 'est donc pas lié à l'articulation d'un contenu, mais au surgissement original d'une altérité qui se tourne vers moi et me regarde: "vocatif» d'une "interpellation derrière la thématisation " ${ }^{69}$. "Visage» sera désormais la qualification de cette étrange "épiphanie » qui déjoue toutes les séductions de l'apparaître pour se révéler "tout entière langage $»^{70}$. Mais langage pris à sa source ou au lieu de son envoi, et qui donne à la raison le visage magistral d'un impératif absolu: «Le langage, c'est la possibilité pour un être d'apparaître du dehors, pour une raison d'être toi, de se présenter comme visage, tentation et impossibilité du meurtre ${ }^{71}$. C'est dans cette «luisance du visage» que perce une "parole» qui «dans son essence est commandement»: qui ne parle pas de quelque chose ou de quelqu'un, mais qui «invite à parler $»^{72}$.

62. «La Signification», p. 371.

63. Ibid., p. 370 .

64. Totalité et Infini parlera donc de "signification sans contexte» (p. XII).

65. "La Philosophie et l'idée de l'Infini», texte de 1957, in En découvrant l'existence avec Husserl et Heidegger, p. 165-178; citation p. 172, dont la formulation est reprise dans Totalité et Infini, p. 170. Ce langage de «l'expérience», central dans Totalité et Infini (p. 43, 46, 81, 170, 194 et passim), sera abandonné par la suite (en raison de ses connotations trop hégéliennes) au profit de termes comme «intrigue» ou «épreuve».

66. «La Signification», p. 376.

67. Là est sans doute une différence majeure entre Levinas et Éric Weil. C'est à propos de ce dernier et de sa grande introduction à la Logique de la philosophie que Paul Ricour pose la question: "Qu'est-ce qui nous fait entrer dans le discours plutôt que demeurer dans la violence? ( La Nature et la Règle - Ce qui nous fait penser, Paris, Odile Jacob, 1998, p. 297).

68. "Transcendance et hauteur», conférence de 1962, reprise dans Liberté et commandement, Montpellier, Fata Morgana, 1994, p. 49-100; citation p. 53.

69. Notes philosophiques diverses, liasse C, p. 418.

70. «La Philosophie et l'idée de l'Infini», p. 173.

71. "Parole et Silence», p. 93.

72. «Liberté et commandement» [1953], texte repris dans Liberté et commandement, p. 27-48; citations p. 48 et 42 . Totalité et Infini le redira à sa manière: «Parler, au lieu de "laisser être", sollicite autrui. La parole tranche sur la vision» (p. 169). 
C'est dans et par cette parole parlante, silencieusement parlante, du visage que se révèle la hauteur du sens (éthique) qui juge et ordonne toutes les significations (culturelles). Il y a là une présentation ou une visitation dont l'auto-signifiance excède sans retour toutes les représentations anticipatives de celui à qui elle s'adresse: le Visage est cet énigmatique "donné que je ne me suis pas donné »73. C'est en raison de cette extériorité ou de cette altérité qui n'est pas à ma mesure qu'il peut et doit être reconnu comme portant «l'investiture du Créateur ${ }^{74}$ : son enseignement me révèle une intelligibilité qui «m'est antérieure ${ }^{75}$, sa parole impérative a l'autorité éthique d'une injonction religieuse ${ }^{76}$ qui interdit et ordonne, qui requiert et met à part son destinataire.

\section{L'inéluctable responsabilité de l'appelé}

Vue du côté du «sujet» de la parole, cette expérience d'une interpellation première, irréductible à ses "capacités», atteste que le langage ne nous advient qu'à partir d'une réception liée sans doute à la tradition culturelle dont nous héritons, mais qui la déborde aussi absolument. Impossible d'accéder à la vérité de notre être parlant autrement que sous le mode de la réponse à une provocation qui nous arrive on ne sait d'où. Levinas reprend à cet égard le vocabulaire de la "gloire» (biblique), mais en le référant à cette écoute d'altérité: «La Gloire de la créature ne me vient que de l'Autre, est entendue ${ }^{77}$. Il faut cet affect ou cette touche d'Autrui pour susciter ou (r)éveiller dans le moi un Désir qui «d'emblée pense plus qu'il ne pense ${ }^{78}$, qui se découvre habité et investi par un infini qu'il ne peut contenir ni mesurer, obligé donc de se laisser transporter hors de soi. Précisons quelques traits de cette expérience paradoxale, où c'est justement l'extériorité irréductible de l'Autre qui vient frapper le Même au plus intime de soi en l'assignant à l'infini de la responsabilité.

73. Notes philosophiques diverses, liasse C, p. 443.

74. Ibid., p. 451.

75. «Liberté et commandement», p. 45.

76. Sur cette portée religieuse de la parole magistrale et d'un «dieu qui parle» (Euvres 2, p. 225) ici, cf. notamment les dernières pages (p. 225-229) de la conférence de 1952 sur «L'Écrit et l'Oral», in CEuvres 2, p. 199-229; ainsi que les réflexions de la conférence de 1955 sur «Le Vouloir» qui évoquent la «Philosophie comme spiritualisation de la religion [...], comme métaphysique et comme morale» (p. 255).

77. «Parole et Silence», p. 96.

78. "La Philosophie et l'idée de l'Infini», p. 172. Levinas emprunte évidemment à Descartes ce «débordement de la pensée finie par son contenu» (Totalité et Infini, p. 171), mais en l'interprétant de manière originale, en référence première à Autrui : il s'y agit de «recevoir d'Autrui au-delà de la capacité du fini » (ibid., p. 22) ou de reconnaître que "posséder l'idée de l'Infini, c'est déjà avoir accueilli Autrui» (ibid., p. 66). 
En un premier temps, c'est l'aspect négatif, dérangeant ou déstabilisant, de cette irruption qui se manifeste. L'irruption ne va pas sans interruption, la survenue du Visage est par là même la «mise en question du Moi par l'Autre ${ }^{79}$. Loin de s'identifier simplement à la liberté, la conscience (morale) naît du jugement qui lui interdit non seulement le meurtre, mais tout discours d'auto-justification. Tout se passe comme si l'intentionnalité spontanément impérial(ist)e du sujet se trouvait désarçonnée, comme si son impétuosité de "force qui va" s'éprouvait paralysée et réduite au silence. Devant la nudité et le dénuement du Visage, devant la faiblesse sans recours de sa résistance éthique, «je ne peux plus pouvoir» ${ }^{80}$, je me trouve simplement renvoyé à la honte de ce que je prenais abusivement pour ma gloire: la violence et l'injustice d'une position s'érigeant arbitrairement en centre et mesure de tout.

Cette déposition, cependant, n'est que la face négative d'une injonction positive: le visage de l'autre qui se tourne vers moi en me vidant de mon enlisement en moi en appelle aussi à moi en tant que "pour autrui» ${ }^{81}$, "parlant par excellence; répondant, responsable» ${ }^{82}$. L'interdiction du meurtre est une obligation de parole, une "sommation de répondre» ${ }^{83}$; et le même geste qui «vide le Moi de son impérialisme et de son égoïsme [...] le confirme dans son ipséité, dans sa place centrale dans l'être, support de l'univers ${ }^{84}$. Se tenant ainsi tout entier entre appel (de l'Autre) et réponse (du Moi), le langage est porté par une intentionnalité proprement éthique: «celle de la responsabilité à l'égard de l'Autre » ${ }^{85}$. Il ne s'y agit pas de comprendre (le monde ou même l'autre), mais de répondre (à celui qui s'adresse à moi). Et ce langage de responsabilité ne peut aller sans la mise en

79. «La Métaphore», p. 343. Ce qu'énonce également Totalité et Infini en parlant du «Visage indiscret d'Autrui qui me met en question» (p. 145) dans ma "glorieuse spontanéité de vivant» (p. 56).

80. "La Philosophie et l'idée de l'Infini», p. 173. C'est déjà le langage de «Liberté et commandement», p. 65. Et ce sera encore celui de Totalité et Infini évoquant la paralysie de «mon pouvoir de pouvoir» (p. 172).

81. C’est par la gloire de l'entendre que le sujet «s'est débordé. Son en soi devient un pour autrui» («Parole et Silence», p. 89).

82. "Le Moi et la Totalité», p. 38 ; c'est cet impératif du visage qui rend le même « responsable, c'est-à-dire parlant» (p. 46). Le moi se trouve ainsi renvoyé par l'autre à ce qu'il est de par sa vocation originelle: «dans sa position même, de part en part responsable» («Liberté et commandement», p. 66).

83. «La Métaphore», p. 343.

84. "Liberté et commandement", p. 67. Totalité et Infini dira de manière analogue que, si «le moi est un privilège ou une élection » irrécusable, il faut «l'appel à la responsabilité infinie » pour le confirmer dans son inaliénable "position apologétique» (p. 223).

85. Notes philosophiques diverses, liasse B, p. 351. Totalité et Infini précisera cette orientation en soulignant que c'est «l'urgence de la réponse - pointe aiguë du présent - (qui) m’engendre pour la responsabilité» (p. 153). 
place d'une dissymétrie fondamentale: impossible de répondre justement à l'Autre sans le saluer - sans l'invoquer - dans la dimension de hauteur qui fait de lui mon maître.

Le sens radical du langage, son événement essentiel, se trouve ainsi selon Levinas dans la relation originale de «l'un pour l'autre - l'un en face de l'autre sans l'intermédiaire d'un contenu commun ${ }^{86}$. Le paradoxe fondamental de cette «rencontre» est qu'elle se produit dans une «relation entre absolus " ${ }^{87}$ ou une relation sans relation qui maintient l'extériorité des sujets qu'elle relie: chacun "s'absout de la relation où il se présente, reste absolu dans cette relation ${ }^{88}$. Dans ce monde de la signifiance, Autrui est l'hôte invitant qui «m'y attend et qui m'y appelle " ${ }^{89}$, alors que le moi n'y accède que comme hôte invité et ainsi transporté hors de lui-même. Les interlocuteurs "s'invoquent ${ }^{90}$ sans doute dans une sorte de prière réciproque ${ }^{91}$, mais sans que puissent jamais se confondre le commandement de l'Autre («Écoute!») et la réponse du Même («Me voici!»). Il n’est de vérité de «la» parole qu'éclatée en dialogue(s), dans «une relation entre libertés qui ne se limitent ni ne se nient, mais s'affirment réciproquement ${ }^{92}$. Ce double mouvement, d'abaissement et d'élévation, pourrait bien être le lieu de passage d'une transcendance religieuse: «Mener vers le haut est un mouvement irréductible, le fond de la spiritualité humaine - de l'être qui parle. Je l'appelle religion ${ }^{93}$.

Expérimenté notamment du côté de celui qui se trouve enseigné et ordonné, ce sens religieux de la parole-réponse débouche sur un double paradoxe. 1) Le jugement qui vide le moi de lui-même le renvoie à l'antériorité d'un "passé absolu», soit d'un "profond jadis - "jadis jamais assez" - jamais saisissable - mais un jadis enseigné» 94 : celui de la "créature

86. «Parole et Silence», p. 94. Totalité et Infini reprendra cette «thèse»: «Nous tâcherons de montrer que le rapport du Même et de l'Autre [...] est le langage» (p. 29), en ajoutant que c'est «l'infini (qui) se produit dans (cette) relation du Même avec l'Autre» (p. XIV).

87. Notes philosophiques diverses, liasse A, p. 257.

88. "La Séparation», p. 268.

89. «La Signification", p. 366.

90. «Le Vouloir», p. 253.

91. Qui fait dire à Levinas que «l'essence du discours est prière» («L'ontologie est-elle fondamentale?», texte de 1951, dans Entre nous..., p. 13-24; citation p. 20).

92. "Le Moi et la Totalité», p. 48. C'est cette transcendance réciproque, ajoute Levinas, qui fait que «Nous n'est pas le pluriel d'un Je» (p. 49).

93. Notes philosophiques diverses, liasse A, p. 232. Dès cette époque, on le voit, Levinas aborde cette expérience du langage à partir du sujet appelé à écouter et répondre.

94. "Les Enseignements», p. 185-186. Levinas aimait citer cette formule du Cantique des Colonnes de Valéry pour évoquer «la trace de l'Absent absolument révolu, absolument passé» («La trace de l'Autre» [1963], dans En découvrant l'existence avec Husserl et Heidegger, p. 187202; citation p. 198). 
antérieure à l'homme " 95 , toujours déjà marquée du sceau de la parole et de sa gloire, et qui ne cesse ainsi de se découvrir engagée plus avant dans l'infini d'une responsabilité qui s'accroît en se recevant ${ }^{96}$. 2) Il y a là une simultanéité de l'évidement et du ressourcement ${ }^{97}$ qui atteint le sujet au plus intime de lui-même, mais dans une tangence de l'esprit et de la chair ou de la parole et du corps qui l'oblige à «accueillir le visage de l'infini de toutes les ressources de son égoïsme, économiquement ${ }^{98}$. C'est cette «inscription» du langage dans les articulations de la condition finie qu'il importe pour finir de mettre en relief.

\section{Les partages du parler juste}

À la pointe de sa signifiance, le langage exprime le face-à-face immédiat du même et de l'autre, il est porté par une pure injonction («Écoute!») dont la résonance suscite ou (r)éveille une parole non moins pure de simple réponse ou d'infinie responsabilité («Me voici»). Mais le risque est alors de prendre pour une réalité donnée ce qui n'est qu'une inspiration et de sombrer ainsi dans l'évanescence, voire la contradiction, d'un dialogue ou d'une interlocution vide de tout contenu; alors que «l'enseignement comporte une signification ${ }^{99}$ et qu'il faut bien que «le son» devienne «signe ${ }^{100}$... A A ssi Levinas insiste-t-il sur la nécessité - et sur l'obligation de rendre justice à cet envoi premier du langage en faisant passer son appel dans la réalité ambiguë des significations économiques, mondaines et culturelles, sociales et historiques. C'est de l'écoute même que (re)naît l'invitation à parler autrement, en inscrivant cette parole-réponse dans les défilés contraignants d'un «discours cohérent ${ }^{101}$ ou d'une thématisation heureusement porteuse d'objectivité et d'universalité.

95. Suivant le mot des Notes philosophiques diverses, liasse B, p. 368 .

96. Selon l'explicitation de Totalité et Infini, surtout p. 222. L'article de l'Encyclopaedia Universalis sur «L'Infini », repris dans Altérité et Transcendance, p. 69-89, voit «le dessin formel de la notion d'infini» dans le fait «que l'altérité de l'Infini puisse consister [...] à se faire proximité et responsabilité» toujours accrues et «que le fini soit ainsi», selon une formulation étrangement proche de la spiritualité ignatienne, « comme pour la plus grande gloire de l'Infini» (p. 88-89).

97. Cf. notamment «La trace de l'autre», p. 193: «La relation avec Autrui me met en question, me vide de moi-même et ne cesse de me vider en me découvrant ainsi des ressources nouvelles» d'accueil et d'hospitalité.

98. Totalité et Infini, p. 191.

99. «Parole et Silence», p. 95.

100.Ibid., p. 99.

101. Contre la suffisance rationaliste, Levinas en appelle bien à la liberté, mais à une liberté se laissant provoquer à la raison: «il y a comme une nécessité de persuasion en faveur d'un discours cohérent. Et c'est peut-être cette persuasion, cette raison avant la raison, qui rend humains le discours cohérent, la raison impersonnelle» («Liberté et commandement», p. 36). 
Il faut rappeler, à cet égard, que la parole vive de l'enseignement et de sa réception se produit nécessairement à l'intérieur d'une tradition d'écriture qui risque toujours de se figer en "mythologie ou philologie » ${ }^{102}$ aliénante, «incapable de se porter secours» ${ }^{103}$. La parole qui s'inscrit en elle n'est pas vouée au non-sens, mais elle s'absente inévitablement en "passé absolu » prenant forme de «testament $»^{104}$ à honorer : «Cette parole sans appel [...] parle sans écouter; elle est loi » ${ }^{105}$ en attente d'actualisation. Entre écrit et oral, la parole ne demeure donc vivante qu'en acceptant le travail jamais achevé de l'objectivation et de l'interprétation: «Les livres appellent des livres, mais cette prolifération d'écrits s'arrête ou culmine au moment où la parole vivante s'y insère, où la critique s'épanouit en enseignement. Et puis, de nouveau, il y a des livres ${ }^{106}$. Les cultures et leurs textes sont le lieu de cette parole-en-travail qu'il importe de réveiller et de relancer sans fin pour que le monde qui s'y trouve exprimé et célébré s'ouvre aux expositions et aux propositions, aux entretiens et aux partages seuls susceptibles de le rendre véritablement commun ${ }^{107}$ en jugeant et ordonnant la «sarabande des cultures innombrables et équivalentes ${ }^{108}$.

Du côté des sujets parlants, cela suppose que la proximité dissymétrique du face-à-face débouche sur une co-présence égalitaire des uns avec les autres, entre personnes qui «se dégage(nt) de (leur) position sexuelle» ${ }^{109}$ : la parole «instaure l'ordre intersubjectif ${ }^{110}$ de la civilisation "sous la simultanéité de la fable ${ }^{111}$ sociale. L'enseignement des fils tend ainsi à se prolonger en reconnaissance mutuelle des frères «sur un plan où l'universel et le particulier [...] se concilient $»^{112}$. Levinas soutient même que, de $c e$ point de vue, «La politique [est] au-dessus de la morale»: «La liberté n'est pas héroïque. L'homme moral est donc obligé de créer des institutions où la liberté pourrait s'exercer ${ }^{113}$. Et ce passage du dialogue éthique à la

102. «L'Écrit et l'Oral», p. 204.

103.Ibid., p. 203.

104.Ibid., p. 211.

105.Ibid., p. 212.

106. «Les Enseignements», p. 187.

107. Totalité et Infini dira magnifiquement que «voir le Visage, c'est parler du monde» (p. 149) et que "parler, c'est rendre le monde commun» (p. 49). Thématisation et objectivation se trouvent alors soutenues par l'offrande d'une parole dont la proposition retentit « dans un monde où il faut recevoir et donner» (p. 191).

108. «La Signification», p. 381; formulation identique dans «La Signification et le Sens », p. 55.

109. «Parole et Silence», p. 100.

110.Ibid., p. 104.

111. «Pouvoirs et Origine», p. 149.

112. «Les Enseignements», p. 197.

113. Notes philosophiques diverses, liasse A, p. 265, qui ajoute cependant de manière significative que «l'institution de la cité peut à son tour devenir inhumaine». Totalité et Infini se situera 
discussion politique relève donc en ce sens d'une obligation incontournable: «En aucune façon ce mouvement qui fonde la civilisation ne devrait être considéré comme une dégradation ou une chute» ${ }^{114}$.

La justesse du dire, on le voit, se cherche à égale distance d'une évasion éthérée et d'un enlisement aliénant. Pensant et parlant, l'homme se tient bien à l'intérieur du monde naturel et historique, mais sans se laisser absorber par lui, en faisant résonner en lui une parole qui appelle à une justice faisant droit à tous et à chacun: «le monde humain est un monde où l'on peut juger l'histoire ${ }^{115}$. Le rapport de l'infini et de la totalité ne saurait être d'identification, mais il ne doit sans doute pas non plus se penser sous le mode de la simple opposition polémique, moins encore de l'exclusion: «S'en dégager tout en y accomplissant une œuvre, ce n'est pas se poser contre la totalité, mais pour elle. Servir la totalité, c'est lutter pour la justice ${ }^{116}$. L'histoire justement jugée - et donc désabsolutisée - pourrait être «l'histoire des sorties hors de la totalité ${ }^{117}$ : lieu paradoxal où le corps et la parole s'altèrent mutuellement, permettant à l'idée grecque de totalité et à l'épreuve sémitique de l'infini de se rencontrer, sinon de se réconcilier.

Il est possible de se demander, bien sûr, si ce "retour» à l'immanence économique des significations culturelles et historiques ne réinscrit pas, comme malgré elle, la pensée de Levinas dans le monde d'une totalité trop synchronique et ne lui impose pas une sorte d'assignation définitive «à résidence ontologique ${ }^{118}$. Comme si la préséance indûment accordée au duo de «l'un-pour-l'autre» était contrainte de ne (re)trouver la justice envers tous les autres que sous une forme à la fois trop dérivée et trop universellement égalitaire ${ }^{119} \ldots$ Mais on risque alors d'oublier deux points qui, dès

plus explicitement encore dans le sillage de «la grande méditation de la liberté par Hegel» pour soutenir la nécessité et le sens de cet ordre objectif: «L'intériorité ne peut remplacer l'universalité. La liberté ne se réalise pas en dehors des institutions sociales et politiques qui lui ouvrent l'accès de l'air frais nécessaire à son épanouissement, à sa respiration et même, peut-être, à sa génération spontanée» (p. 218).

114. «Parole et Silence», p. 103. Impossible donc, voire interdit, de faire de la juste égalité politique un dérivé secondarisé de la proximité éthique: «Le tiers me regarde dans les yeux d'autrui. Le langage est justice» (Totalité et Infini, p. 188).

115. «Le Moi et la Totalité», p. 44.

116.Ibid., p. 49.

117. J. Derrida, «Violence et métaphysique», texte de 1964, repris dans L'écriture et la différence, Paris, Seuil, 1967, p. 117-228; citation p. 173.

118. D. Franck, L'un-pour-l'autre, Levinas et la signification, Paris, PUF, 2008. Paul Ricœur avait une position plus nuancée en parlant d' "une quasi-ontologie qu'on peut dire post-éthique " (Autrement, Lecture d'Autrement qu'être ou au-delà de l'essence d'Emmanuel Levinas, Paris, PUF, 1997, p. 35).

119. J'évoque trop schématiquement les objections développées par Didier Franck dans le livre précédemment cité. Je les ai discutées de manière plus précise dans «Résonances 
les années d'élaboration de Totalité et Infini, revêtent sans doute une égale importance pour Emmanuel Levinas: 1) La mise en relief du face-à-face en son exceptionnelle originalité ne rétablit aucun principe hiérarchique, mais se contente de souligner le sens unique du «sujet» en sa position de créature appelée à la responsabilité. 2) La justice à laquelle il est alors fait «retour» relève par là même d'une bonté affectée de proximité, et l'égalité effective qu'elle exige passe par une ouverture à l'infini qui, à travers la mise en commun du monde, ne cesse d'incliner les uns vers les autres les visages toujours singuliers. Tout cet «ensemble» obéit donc à une cohérence paradoxale qu'il vaut sans doute la peine de souligner pour terminer.

\section{Un discours toujours relancé}

À travers les nombreux essais, inédits ou publiés, qui séparent Le Temps et l'Autre (1948) du maître livre qu'est Totalité et Infini (1961), on peut voir s'esquisser une phénoménologie de l'être parlant dont l'intention de cohérence (dialectique) est indéniable. Le centre en est évidemment l'éclat d'une parole enseignante qui enjoint son destinataire à l'infini de la responsabilité. Mais ce Sinaï du face-à-face parlant ne peut être abordé qu'à partir de l'expérience première de la séparation psychique telle qu'elle tend toujours à être absorbée ou résorbée dans les discours de l'immanence ontologique. Il faut laisser l'événement de l'interpellation surgir à l'intérieur de cela même qu'il met en question pour que se (r)éveillent, en nous et entre nous, le goût et l'exigence de dialogues raisonnables chargés de nous rendre le monde plus justement commun. Se trouve ainsi déjà dessinée, au moins en ébauche ou en anticipation «diachronique», l'architecture signifiante de Totalité et Infini, où la parole magistrale du Visage s'arrache aux ambiguités des significations économiques pour ouvrir les interlocuteurs à la responsabilité du monde partagé.

Il y a là un effort toujours recommencé d'articulation, d'ajointement, voire de systématicité raisonnable; mais qui ne cesse précisément de se laisser ouvrir et porter au-delà de lui-même. Déjà sans doute, et de manière décisive, par l'accueil du Visage faisant advenir « un monde où l'on parle et dont on parle ${ }^{120}$, éclairé par la grâce d'une parole qui «est amitié et hospitalité» ${ }^{121}$. Mais le risque demeure d'une civilisation aux pouvoirs "fabuleux", rêvant de s'accomplir dans son présent en se comprenant «à partir de la fable qui

interrogatives», article paru dans Reflections on Levinas, V. Celebi (éd.), Istanbul, Mono Kurgusuz Labirent, 2010, p. 35-51.

120. Totalité et Infini, p. 156.

121.Ibid., p. 282, pour qui c'est là «l'essence du langage». 
par le récit historique arrête son histoire» et " "peut" sur l'avenir» ${ }^{122} \ldots$ On comprend donc que Levinas, en 1959, reprenne ses méditations antérieures sur «l'au-delà du possible» ${ }^{123}$ et le temps de la fécondité où «l'être se libérant de soi - infiniment recommence, c'est-à-dire infiniment est ${ }^{124}$. Comme s'il fallait renvoyer l'altérité éthique du Visage à une autre altérité qui la précède et qui l'excède - autrement: la "merveille humaine de la famille» ${ }^{125}$ où se dessine, à travers le discontinu des générations, "ce temps de la bonté qui est au-delà du possible ${ }^{126}$. Et Totalité et Infini cherchera à penser dans la trace signifiante du Visage le recueillement d'une intimité qui l'accueille et le porte finalement «au-delà » de lui-même, à travers l'unicité plurielle d'une élection à la fois filiale et fraternelle ${ }^{127}$; éclairées par la parole, la rencontre et la fécondité des corps permettent d'annoncer "à nos frères un engendrement, à nos fils une fraternité ${ }^{128}$.

Le chemin de pensée de Levinas ne s'arrêtera pas avec Totalité et Infini, pas plus d'ailleurs qu'avec Autrement qu'être ou De Dieu qui vient à l'idée... Mais toutes les nouveautés éminemment signifiantes de ce discours toujours relancé (trace, illéité, Dire, substitution, (r)éveil, etc.) témoigneront toujours, à travers une exposition jamais satisfaite de ses élaborations raisonnables, d'une dimension d'altérité excessive, anarchique et imprenable, qui habite et traverse les relations interhumaines, que le logos ne saurait maîtriser, mais par laquelle il lui revient de se laisser provoquer toujours à nouveau. La probité philosophique «annonce l'avenir» qui nous attire et nous appelle, «mais ne l'accomplit pas» ${ }^{129}$.

Francis GUIBAL

Université de Strasbourg

122. «Parole et Silence», p. 104. La «fable» renvoie sans doute implicitement à la «fonction fabulatrice» analysée par Bergson dans Les deux sources de la morale et de la religion. Et «l'arrêt de l'histoire» pourrait bien faire allusion à l'interprétation de Hegel par Kojève...

123. «Au-delà du possible», in Euvres 2, p. 291-318.

124.Ibid., p. 303; on mesure la distance qui sépare encore cet «infiniment être» de «l'autrement qu'être " s'arrachant, en se dédisant, à tout "être autrement " (Autrement qu'être ou au-delà de l'essence, La Haye, Martinus Nijhoff, 1974, p. 8).

125. «Au-delà du possible», p. 311.

126.Ibid., p. 312. Sur cette diachronie qui porte l'être à l'infini d'un temps toujours recommencé, je me permets de renvoyer au chapitre 1, "L'étrange aventure de la paternité», de mon ouvrage Emmanuel Levinas - Le sens de la transcendance, Autrement, Paris, PUF, 2009, p. $15-29$.

127. C'est l'ensemble de la quatrième et dernière partie du livre qui, sous le titre global «Au-delà du visage», suit la dialectique de la fécondité amoureuse à travers l'infini du temps de la fécondité.

128. P. Beauchamp, Psaumes nuit et jour, Paris, Seuil, 1980, p. 232 (à propos du psaume 22).

129. «Parole et Silence», p. 104. 